\title{
Chromosomal Mapping of Repetitive DNAs in Myiopsitta monachus and Amazona aestiva (Psittaciformes, Psittacidae) with Emphasis on the Sex Chromosomes
}

\author{
Ivanete de Oliveira Furo ${ }^{a, c}$ Rafael Kretschmer ${ }^{d}$ Michelly S. dos Santos ${ }^{\text {a, } c}$ \\ Carlos A. de Lima Carvalho ${ }^{a, c}$ Ricardo J. Gunski ${ }^{\text {e Patrícia C.M. O’Brien }}{ }^{9}$ \\ Malcolm A. Ferguson-Smith ${ }^{9}$ Marcelo B. Cioffi ${ }^{f}$ Edivaldo H.C. de Oliveira ${ }^{b, c}$ \\ a Programa de Pós Graduação em Genética e Biologia Molecular, and b Faculdade de Ciências Naturais, Instituto de \\ Ciências Exatas e Naturais, Universidade Federal do Pará, Belém, ' Laboratório de Cultura de Tecidos e Citogenética, \\ SAMAM, Instituto Evandro Chagas, Ananindeua, dPrograma de Pós Graduação em Genética e Biologia Molecular, \\ Universidade Federal do Rio Grande do Sul, Porto Alegre, ePrograma de Pós Graduação em Ciências Biológicas, \\ Universidade Federal do Pampa, Campus São Gabriel, São Gabriel, and f Departamento de Genética e Evolução, \\ Universidade Federal de São Carlos, São Carlos, Brazil; ${ }^{9}$ Cambridge Resource Centre for Comparative Genomics, \\ Cambridge, UK
}

\section{Keywords}

Constitutive heterochromatin · Karyotype description ·

Microsatellites · Neotropical Psittacidae · Sex chromosomes

\begin{abstract}
Here, for the first time, we describe the karyotype of Myiopsitta monachus (Psittacidae, Arini). We found $2 n=48$, corresponding to the lowest diploid number observed in Neotropical Psittaciformes so far, with an uncommonly large W chromosome homomorphic to the Z. In order to better understand the evolution of the sex chromosomes in this species, we applied several molecular cytogenetic approaches, including C-banding, FISH mapping of repetitive DNAs (several microsatellite repeats), and whole-chromosome painting on metaphases of $M$. monachus. For comparison, another species belonging to the same tribe but with a smaller W chromosome (A. aestiva) was also analyzed. The
\end{abstract}

results show that the constitutive heterochromatin has a very diverse distribution pattern in these species revealing heterochromatic blocks in the centromeric region of all chromosomes and in most of the length of the $\mathrm{W}$ chromosome in A. aestiva, while in M. monachus they were found in interstitial and telomeric regions. Concerning the microsatellites, only the sequence $(C G)_{n}$ produced signals on the $W$ chromosome of $A$. aestiva, in the distal region of both arms. However, in M. monachus, $(C A A)_{n},(C A G)_{n}$, and $(C G)_{n}$ probes were accumulated on the $\mathrm{W}$ chromosome, and, in addition, the sequence $(C A G)_{n}$ also hybridized to heterochromatic regions in macrochromosomes, as well as in microchromosomes. Based on these results, we suggest that the increase in length of the $\mathrm{W}$ chromosome in $\mathrm{M}$. monachus is due to the amplification of repetitive elements, which highlights their significant role in the evolutionary process of sex chromosome differentiation.

(c) 2017 S. Karger AG, Basel

\section{KARGER}

(C) 2017 S. Karger AG, Basel

E-Mail karger@karger.com

www.karger.com/cgr
Dr. Edivaldo Herculano C. de Oliveira

Laboratório de Cultura de Tecidos e Citogenética - SAMAM

Instituto Evandro Chagas, BR $316 \mathrm{KM} 7, \mathrm{~s} / \mathrm{n}$ - Levilândia

Ananindeua, PA 67000-030 (Brazil)

E-Mail ehco@ufpa.br, ehco@uol.com.br 
Table 1. Species and number of samples analyzed in this study

\begin{tabular}{llll}
\hline Species & Individuals, $n$ & Sex & Place of collection \\
\hline Amazona aestiva & 2 & $2 \%$ & Museu Paraense Emílio Goeldi, Belém, Pará \\
Myiopsitta monachus & 3 & $2 \uparrow, 10^{7}$ & $\begin{array}{l}\text { Parque Zoológico do Rio Grande do Sul, Sapucaia, } \\
\text { Rio Grande do Sul }\end{array}$ \\
\hline
\end{tabular}

Neotropical parrots (family Psittacidae) are one of the most threatened groups of birds in the world, due to environmental factors such as deforestation, resource extraction, and also illegal trapping and nest poaching for the pet trade [Wright et al., 2001; Laurance et al., 2002]. However, little is known about many of their biological aspects, such as population genetic structure, genome evolution, and phylogenetic relationships [Wright et al., 2001; Presti and Wasko, 2014]. Considering the scarce information about their genome organization, it is known that Neotropical Psittacidae have a very homogeneous diploid number, with $2 \mathrm{n}=70$ in most species, with only few exceptions, such as Graydidascalus brachyurus $(2 \mathrm{n}=64)$ [Caparroz and Duarte, 2004]. Regarding the sex chromosomes, all species present the typical ZW sex chromosome system found among birds, where the $\mathrm{Z}$ chromosome usually is a metacentric element, corresponding in size to the $3 \mathrm{rd}$ or 4 th pair, while the $\mathrm{W}$ varies morphologically (metacentric, submetacentric, or telocentric) and has a size similar to the smallest macrochromosome pairs [Francisco and Galetti, 2001; Santos and Gunski, 2006].

Recently, a genomic-scale comparison of 48 bird species revealed that avian genomes have only a small amount of repetitive DNAs (4-10\%), a value smaller than that one found in other tetrapods (e.g., $34-52 \%$ in mammals) [Zhang et al., 2014]. Some authors suggested that the small size of the bird genomes represented an adaptation to the high rate of oxidative metabolism resulting primarily from the demands of flight. Thus, nonflying birds have bigger genomes than flying ones [Wachtel and Tiersch, 1993; Hughes and Hughes, 1995; Hughes, 1999; Venton, 2012]. An exception to this rule was found in the downy woodpecker (Picoides pubescens), a species belonging to the order Piciformes, which has about $22 \%$ of repetitive DNA sequences, resulting from an accumulation of sequences like LINE (long interspersed elements) type CR1 (chicken repeat 1) transposons [Zhang et al., 2014]. This may also be the case for Psittacidae species which show a higher content of repetitive DNAs evidenced by C-banding, as in Alisterus scapularis [Christidis et al., 1991] and
G. brachyurus [Caparroz and Duarte, 2004], which have an accumulation of heterochromatin in their enlarged $\mathrm{W}$ chromosomes. Hence, although repetitive sequences represent a small percentage of avian genomes, it seems that they play an important role in the differentiation of sex chromosomes, as observed in many other groups [Marchal et al., 2004; Kejnovsky et al., 2009; Cioffi et al., 2011a; Matsubara et al., 2016]. As an example, a recent analysis of sex chromosomes in Sauropsida (reptiles and birds) demonstrated that the amplification of microsatellite repeats was strongly associated with the process of differentiation and heterochromatinization of sex-specific chromosomes [Matsubara et al., 2016].

It is assumed that sex chromosomes have evolved from a pair of homologous autosomes and, during this process, the reduced or absent recombination events were crucial for the accumulation of large amounts of repetitive sequences in the $\mathrm{Y}$ or $\mathrm{W}$ chromosomes [Charlesworth et al., 2005; Zhou et al., 2014; Schartl et al., 2016]. In addition, although $W$ chromosomes are usually smaller than Zs in most ZW species, in some groups of vertebrates, the opposite scenario was observed, as in some lizards and fishes, due to a series of amplifications of repetitive elements [Galetti et al., 1995; Ezaz et al., 2009; Schartl et al., 2016]. Indeed, the accumulation of repetitive sequences, like retrotransposons and microsatellites in sex chromosomes, has been reported in many species of plants and animals [Kraemer and Schmidt, 1993; Hobza et al., 2006; Cioffi and Bertollo, 2012; Ezaz et al., 2013; Ezaz and Deakin, 2014].

However, although the importance of repetitive sequences in sex chromosome evolution has been extensively studied in fishes, as many species show the absence of heteromorphic sex chromosomes [Cioffi et al., 2011a, b], there is only 1 report concerning this topic in avian species in which the pattern of microsatellite distribution in chicken (Gallus gallus) chromosomes was compared to some lizard species [Matsubara et al., 2016]. Hence, the role of repetitive sequences in the process of sex chromosome differentiation in avian species is still poorly understood.
152

Cytogenet Genome Res 2017;151:151-160 DOI: $10.1159 / 000464458$ de Oliveira Furo et al. 
Although the present study was conceived in order to describe the karyotype of the monk parakeet (Myiopsitta monachus) for the first time, the observation of a prominent large $\mathrm{W}$ chromosome in this species prompted us to analyze the possible role of repetitive sequences in the differentiation process of this chromosome. Here, we compare the distribution of several microsatellite repeats in the genome of M. monachus with the results obtained in the blue-fronted amazon, Amazona aestiva, a species with a typical Psittacidae karyotype, with $2 \mathrm{n}=70$ and a smaller W chromosome. We applied several molecular cytogenetic approaches, including C-banding, FISH with repetitive DNA probes, and whole-chromosome painting. The results demonstrate that the increased length of the $\mathrm{W}$ chromosome in M. monachus is caused by the amplification of repetitive elements, highlighting their significant role in the evolutionary process of sex chromosome differentiation.

\section{Materials and Methods}

\section{Samples and Tissue Cultures}

Metaphases were obtained from fibroblast cultures of skin and feather pulp biopsies of 2 individuals of A. aestiva and 3 of M. monachus (Table 1), following Sasaki et al. [1968] with modifications. Samples were fractionated mechanically in Petri dishes, and afterwards incubated in collagenase solution $(0.0186 \mathrm{~g}$ in $4 \mathrm{~mL}$ of DMEM medium), for $1 \mathrm{~h}$ at $37^{\circ} \mathrm{C}$ for cell dissociation. After centrifugation and discarding the supernatant, $5 \mathrm{~mL}$ of DMEM supplemented with antibiotics and fetal bovine serum (10\%) were added, and the material was transferred to culture flasks. Chromosomes were obtained after adding $100 \mu \mathrm{L}$ colcemid $(0.05 \mu \mathrm{g} / \mathrm{mL})$, followed by hypotonic treatment with $0.075 \mathrm{M} \mathrm{KCl}$ for $15 \mathrm{~min}$ at $37^{\circ} \mathrm{C}$, and washes/fixation in Carnoy fixative (methanol:acetic acid 3:1).

Conventional Cytogenetics: Giemsa Staining and Chromosome Banding

The distribution of heterochromatic blocks was analyzed by Cbanding [Sumner, 1972], with modifications. After incubation of the slides at $60^{\circ} \mathrm{C}$ for $1 \mathrm{~h}$, they were treated with $0.2 \mathrm{~N} \mathrm{HCl}$ for 15 $\min , 50 \% \mathrm{Ba}\left(\mathrm{OH}_{2}\right)$ for $15 \mathrm{~min}$ at $37^{\circ} \mathrm{C}, 0.01 \mathrm{~N} \mathrm{HCl}$ for $2 \mathrm{~min}, 2 \times$ SSC at $60^{\circ} \mathrm{C}$ for $1 \mathrm{~h}$, and stained with Giemsa for $25 \mathrm{~min}$. G-banding followed the protocols of Seabright [1971] with modifications. 3-4-day-old slides were used for G-banding. They were incubated in $0.25 \%$ trypsin for $5 \mathrm{~s}$ at room temperature, then briefly immersed in $0.5 \times \mathrm{SSC}$ at $60^{\circ} \mathrm{C}$, and stained with Wright (Merck) for $2 \mathrm{~min}$ and $30 \mathrm{~s}$. Metaphases were analyzed and captured using GenASIs software (Applied Spectral Imaging).

Fluorescence in situ Hybridization

FISH experiments with microsatellites followed Kubat et al. [2008], with slight modifications, and probes were directly labeled with Streptavidin-Cy3. The sequences were $d(C A)_{n}, d(C A A)_{n}$, $\mathrm{d}(\mathrm{CAG})_{\mathrm{n}}, \mathrm{d}(\mathrm{GC})_{\mathrm{n}}$, and $\mathrm{d}(\mathrm{CAT})_{\mathrm{n}}$. We also applied telomeric probes

Mapping of Repetitive DNAs in

M. monachus and A. aestiva

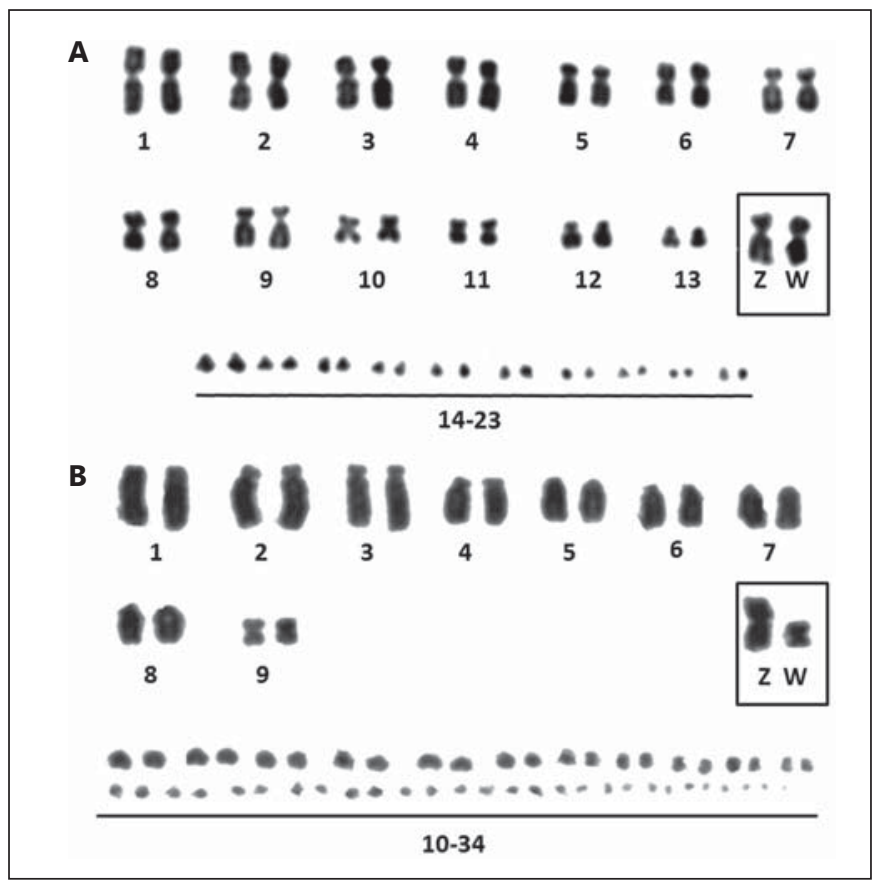

Fig. 1. Giemsa-stained karyotypes of Myiopsitta monachus, $2 \mathrm{n}=$ 48 (A) and Amazona aestiva, $2 \mathrm{n}=70$ (B).

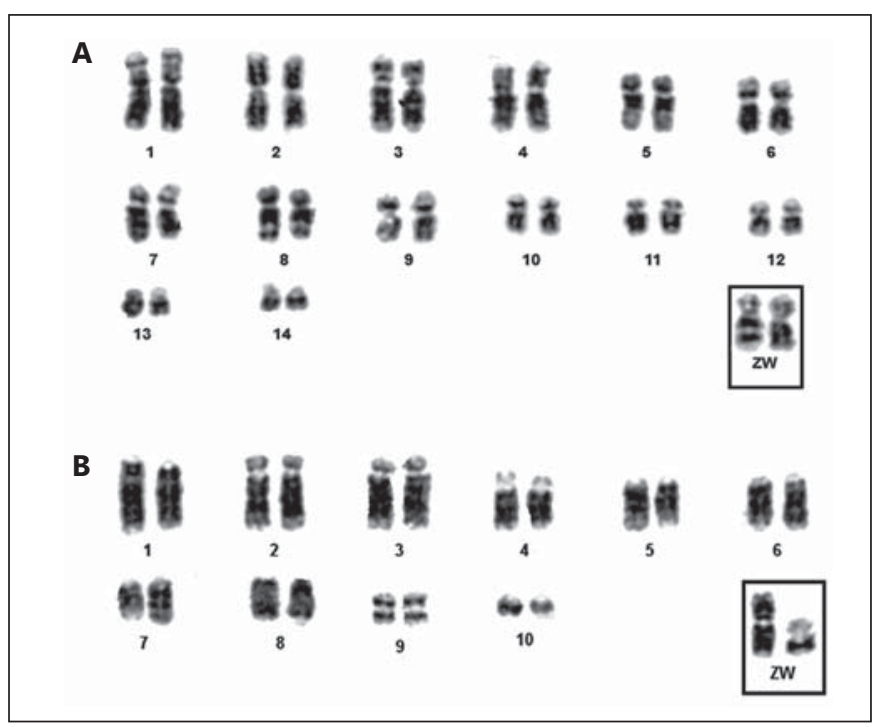

Fig. 2. Partial G-banded karyotypes of a female Myiopsitta monachus (A) and a female Amazona aestiva (B).

generated by PCR in the absence of a template, using (TTAGGG) and (CCCTAA $)_{5}$ as primers [Ijdo et al., 1991]. In addition, for the correct identification of the sex chromosomes, we used a wholechromosome painting probe of the chicken $\mathrm{Z}$ chromosome (GGAZ) following de Oliveira et al. [2005]. This probe was ob-

Cytogenet Genome Res 2017;151:151-160 153 
Fig. 3. C-banded metaphases of a male $M y$ iopsitta monachus (A) and a female Amazona aestiva (B). C Idiogram of C-banded M. monachus chromosomes showing heterochromatin regions in autosomes 1-9 and the sex chromosomes.

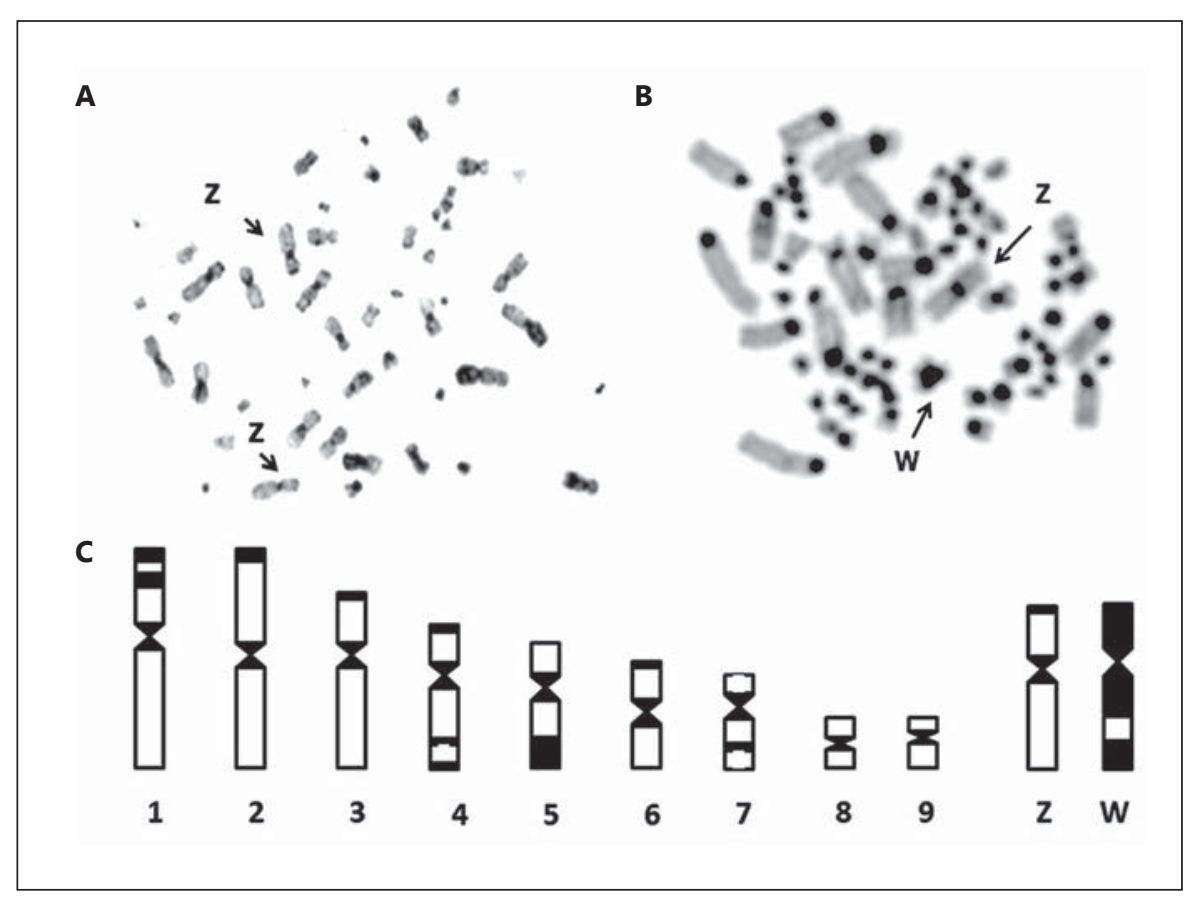

tained according to Griffin et al. [1999]. Images were acquired using a $63 \times$ immersion objective on a Zeiss Imager2 fluorescent microscope and analyzed with AxioVision 4.8 software (Zeiss, Germany).

\section{Microscopic Analyses}

At least 30 metaphase spreads per individual were analyzed to confirm the $2 \mathrm{n}$ and the karyotype structure. Images were captured using a 100× objective (Leica DM1000) and GenASIs software. Chromosomes were classified as metacentric, submetacentric, acrocentric, and telocentric according to their arm ratios [Guerra, 2004].

\section{Results}

\section{Conventional Cytogenetics}

M. monachus revealed the lowest diploid chromosome number described for a South-American Psittacidae species so far, $2 \mathrm{n}=48$, with 28 macrochromosomes, including the sex pair, and 20 microchromosomes. Autosomes 2 and 6 are metacentric, while the other macrochromosomes are submetacentric. $\mathrm{Z}$ and $\mathrm{W}$ sex chromosomes are submetacentric and of similar size, corresponding to the third largest pair (Fig. 1A). Concerning $A$. aestiva, the karyotype was similar to that previously described, with $2 \mathrm{n}=70$. Autosomes $1,5,6,7$, and 8 are telocentric, pairs 2 and 4 are submetacentric, 9 is metacentric, and 3 and 10 are acrocentric. $\mathrm{Z}$ and $\mathrm{W}$ sex chromosomes are metacen- tric and differ in size, corresponding to chromosomes 4 and 9, respectively [Aquino and Ferrari, 1990; Duarte and Caparroz, 1995] (Fig. 1B). The G-banding patterns of the macrochromosomes and the sex chromosomes of both species are shown in Figure 2A and B.

Blocks of constitutive heterochromatin were detected in the autosomes and sex chromosomes in M. monachus. Autosomes 2, 3, 6, and the $\mathrm{Z}$ chromosome showed centromeric and telomeric blocks, while in pairs 1 and 4 interstitial heterochromatic blocks were present. Chromosomes 5 had a large heterochromatin block in the long arms, and chromosome 7 displayed just an interstitial band in the long arms. Although $\mathrm{Z}$ and $\mathrm{W}$ chromosomes are homomorphic, their heterochromatic content is different, with most of the $\mathrm{W}$ being heterochromatic (Fig. 3A, C).

In A. aestiva, heterochromatin is present in the centromeric regions of the macrochromosomes and in the $\mathrm{Z}$ chromosome. In addition, almost all microchromosomes were strongly stained by C-banding. The W chromosome stood out from the other macrochromosomes by showing a larger amount of constitutive heterochromatin (Fig. 3, 5).

\section{Fluorescence in situ Hybridization}

Two of the 5 microsatellite sequences used, $(C A T)_{n}$ and $(\mathrm{CA})_{\mathrm{n}}$, did not produce signals in any of the species. The $(\mathrm{GC})_{\mathrm{n}}$ sequence showed signals only in the telomeric 


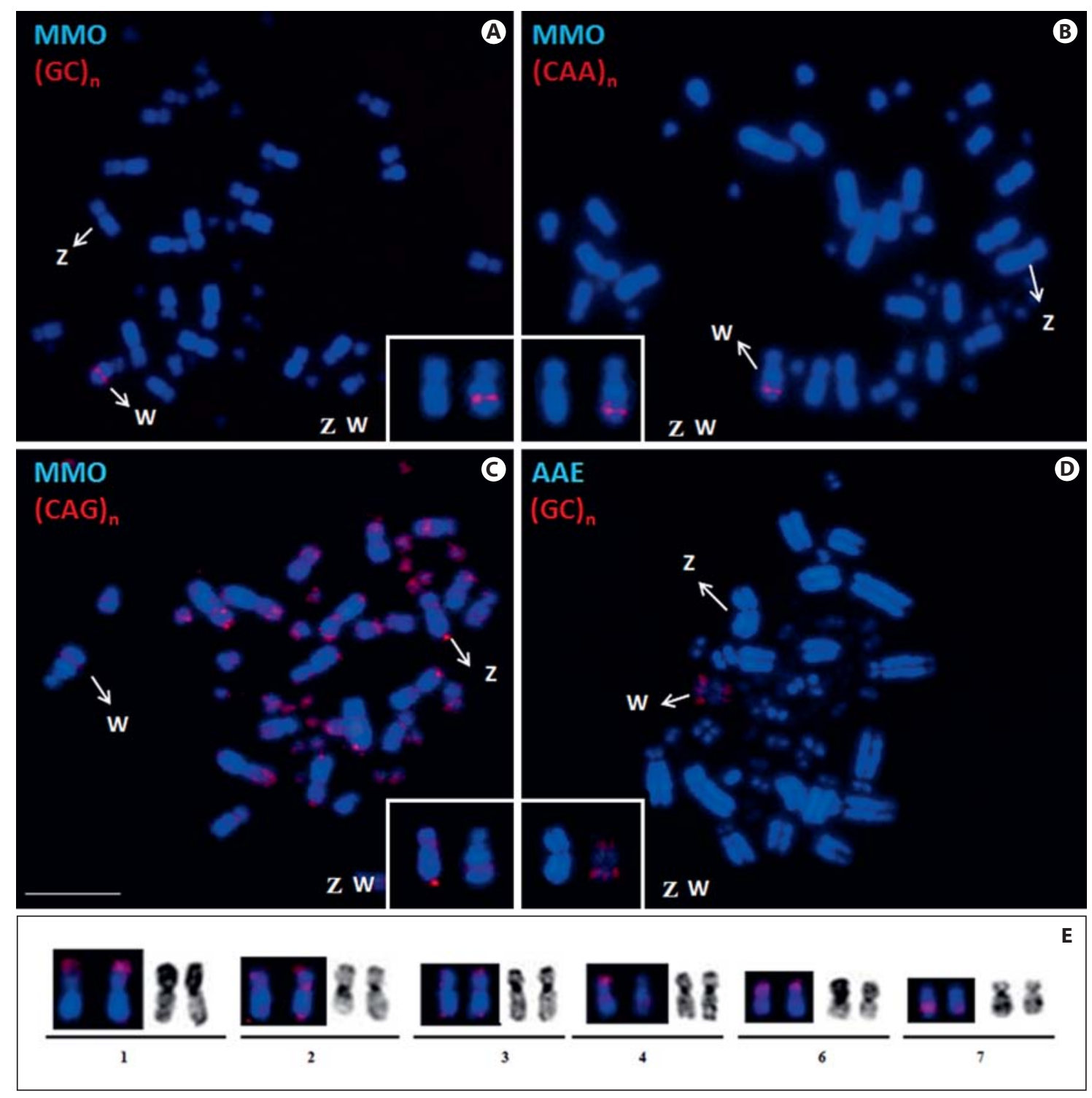

Fig. 4. FISH of microsatellite sequences on chromosomes of Myiopsitta monachus (A-C) and Amazona aestiva (D). A, D $(\mathrm{GC})_{\mathrm{n}}$. B $(\mathrm{CAA})_{\mathrm{n}}$. C $(\mathrm{CAG})_{\mathrm{n}}$. E Heterochromatin blocks which correspond to sequence $(\mathrm{CAG})_{\mathrm{n}}$ in $M$. monachus. Scale bar, $5 \mu \mathrm{m}$.

region of Wq in A. aestiva (Fig. 4D). In M. monachus, the $(\mathrm{CAG})_{\mathrm{n}}$ sequence hybridized in many regions of the macrochromosomes, corresponding to C-positive heterochromatin, and in all microchromosomes. In addition, this sequence hybridized in the sex chromosomes - in the long arm of the $\mathrm{W}$ chromosome and in the short arm of the $\mathrm{Z}$ (Fig. $4 \mathrm{C}) .(\mathrm{GC})_{\mathrm{n}}$ and $(\mathrm{CAA})_{\mathrm{n}}$ probes also hybridized in the long arm of the $\mathrm{W}$ chromosome (Fig. 4A, B). Interestingly, the heterochromatin blocks correspond to the hybridization pattern of $(\mathrm{CAG})_{\mathrm{n}}$ in M. monachus (Fig. 4E).
The identification of the $\mathrm{Z}$ sex chromosome was confirmed by FISH using the GGAZ painting probe, which hybridized only on the $\mathrm{Z}$ chromosome in both species (Fig. 5). However, there were no signals in the distal region of $\mathrm{Zp}$ of both species (Fig. 5).

Telomeric repeats (TTAGGG) produced signals at the ends of the chromosome arms in both species. Moreover, the existence of interstitial telomeric sequences (ITSs) in the $\mathrm{Z}$ chromosome and in pair 2 of A. aestiva became obvious (Fig. 6). 


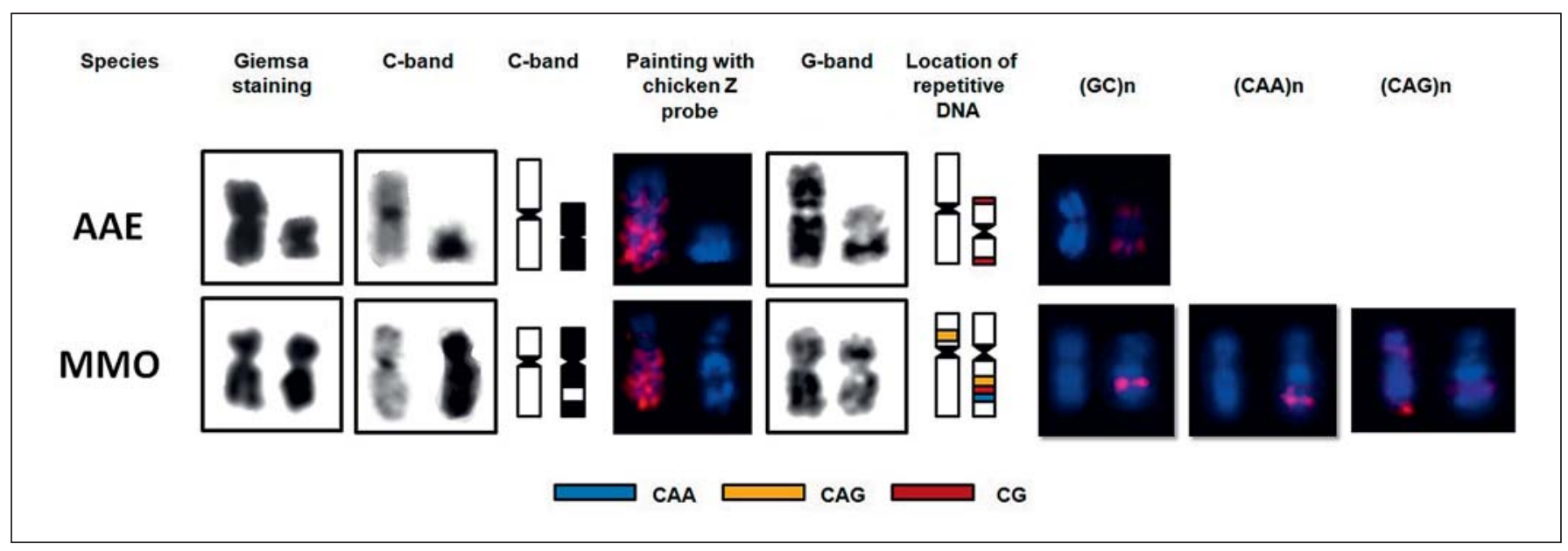

Fig. 5. Summary of Giemsa-staining, C- and G-band patterns, and chromosome painting with GGAZ in the sex chromosomes, and the location of 3 microsatellite sequences on the W chromosomes of Myiopsitta monachus (MMO) and Amazona aestiva (AAE).

Fig. 6. FISH with telomeric probes in metaphases of Amazona aestiva (A) and Myiopsitta monachus (B). Scale bars, $5 \mu \mathrm{m}$.

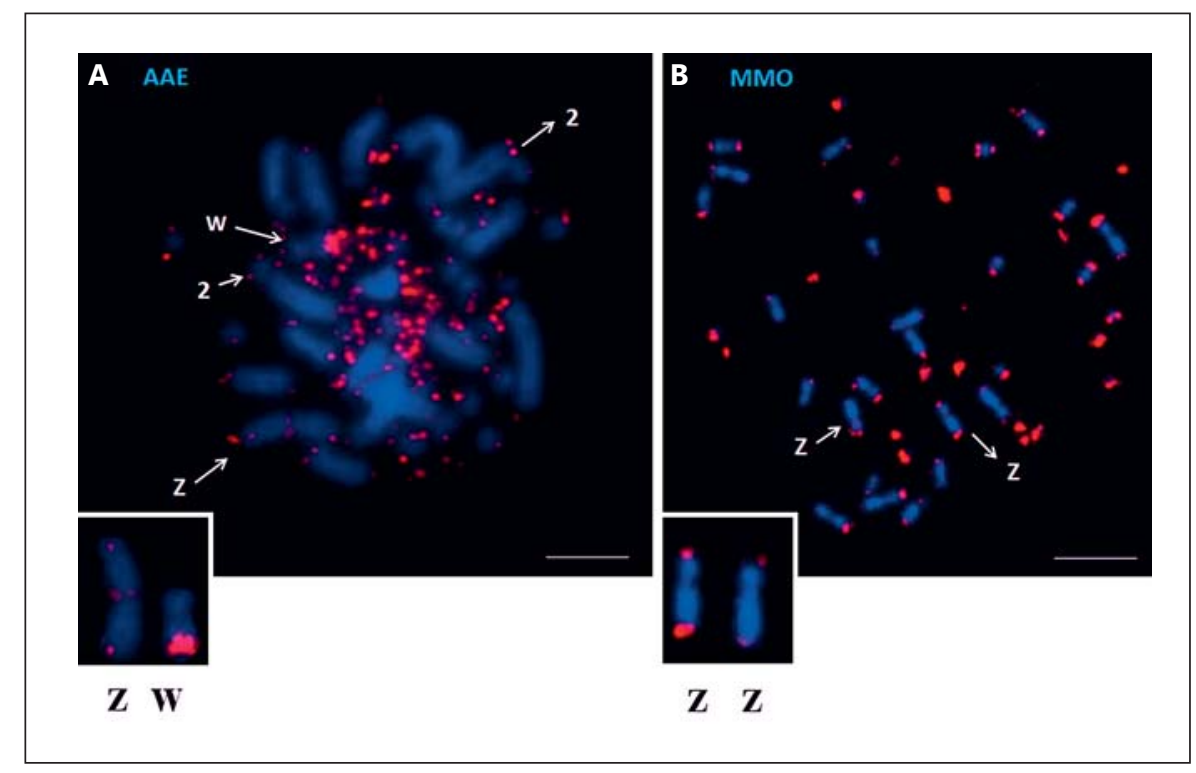

\section{Discussion}

\section{Karyotype Description}

Although most Neotropical Psittacidae exhibit a similar karyotype, both in diploid number $(2 \mathrm{n}=70)$ and chromosome morphology [Van Dongen and Boer, 1984], cytotaxonomic analysis suggests a dichotomy among the representatives of the tribe Arini [Lunardi et al., 2003]. The species of this tribe show 2 basic karyotype patterns: predominantly metacentric and submetacentric macrochromosomes in the genera Ara, Cyanopsitta, Propyrrhura, Aratinga, Pionites, Pionopsitta, Nandayus, and Guaruba [de Lucca and de Marcod, 1983; Duarte and Giannoni, 1990; Goldschmidt et al., 1997; Lunardi et al., 2003], whereas in the genus Amazona, chromosomes 1, 5, 6, and 7 are telocentric, pairs 2 and 3 vary from submetacentric to acrocentric, pair 4 is submetacentric, pair 8 telocentric or metacentric, and pair 9 metacentric [Duarte and Caparroz, 1995], exactly as observed in A. aestiva analyzed here.

In $M$. monachus, we found a chromosome complement differing from the Neotropical species already analyzed so far, with an extremely low diploid number $(2 \mathrm{n}=$ 48 ). Concerning the morphology of the macrochromosomes, in M. monachus, the presence of biarmed macro- 


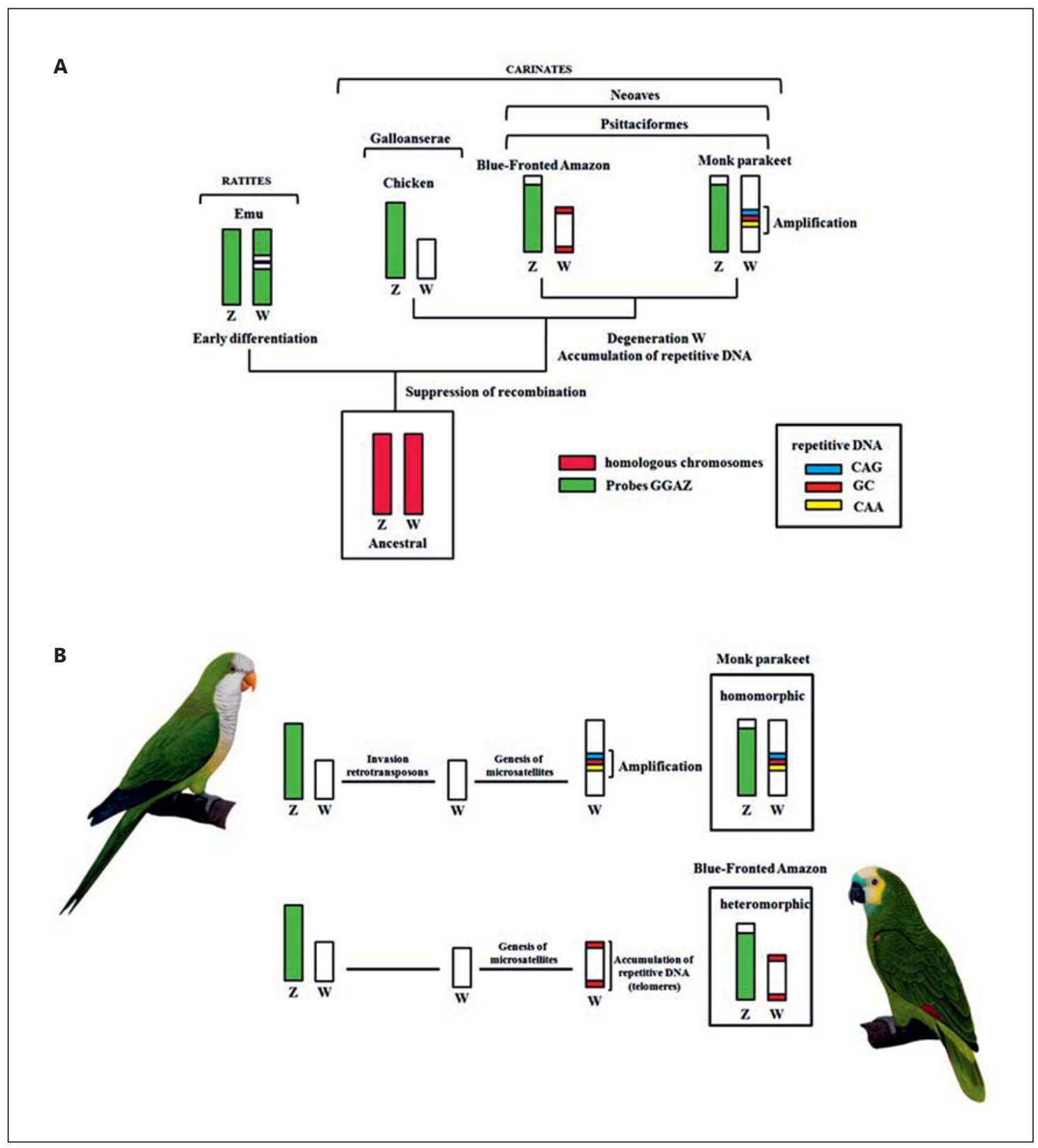

Fig. 7. Evolution of the $\mathrm{W}$ chromosome in Amazona aestiva and Myiopsitta monachus, inferred from the data obtained by FISH with microsatellite sequences. A Early stage of sex chromosome differentiation in Aves, showing the importance of recombination suppression in the degeneration and accumulation of repetitive sequences on the $\mathrm{W}$ chromosome of Neoaves. B Hypothesis for the size variation of the $\mathrm{W}$ chromosome between the 2 species of Neotropical Psittacidae.

chromosomes resembles the patterns observed in some Neotropical Psittacidae, although pairs 1,2, and 6 are metacentric, submetacentric, and acrocentric, respectively, in the long-tailed species analyzed so far, while in $M$. monachus pair 1 is submetacentric, and pairs 2 and 6 are metacentric (Fig. 1A).

Mapping of Repetitive DNAs in M. monachus and A. aestiva
The diploid number of $M$. monachus represents the lowest one for Neotropical Psittacidae analyzed so far. However, there is an African Psittacidae species, Agapornis roseicollis with the same $2 \mathrm{n}$, but with some differences in chromosome morphology. For instance, in A. roseicollis, autosomes 1-7 vary from metacentric to submetacen- 
tric, and pairs 9-11 are telocentric [Nanda et al., 2007], while in $M$. monachus, pairs 1-11 are all biarmed. Besides, the predominance of biarmed elements in M. monachus suggests that fusions may have played an important role in the divergence of this karyotype in relation to other Neotropical Psittacidae. However, this type of rearrangement was not considered in the proposal of Christidis et al. [1991], who postulated only inversions and translocations as the main rearrangements in the karyotype evolution of Psittaciformes.

\section{Heterochromatin Distribution and Telomeric}

Sequences

Like the majority of birds, Neotropical Psittacidae demonstrate a pattern of heterochromatin distribution limited to the centromeric regions, according to data obtained in the genera Aratinga, Forpus, Pionus, Graydidascalus, and Amazona [Aquino and Ferrari, 1990; de Lucca et al., 1991; Goldschmidt et al., 1997; Caparroz and Duarte, 2004]. On the other hand, the distribution of heterochromatic blocks in M. monachus differs from that in other members of the tribe Arini, since C-positive heterochromatic bands were detected in telomeric, interstitial, and centromeric regions in this species. This unique characteristic resembles the results observed in some Cacatuidae species, in which heterochromatic blocks were found also in interstitial regions [Christidis et al., 1991].

Furthermore, the microsatellite repeat $(\mathrm{CAG})_{\mathrm{n}}$ hybridized in many heterochromatin blocks in M. monachus, corroborating the view that repetitive DNAs tend to concentrate in those genome areas of many organisms [Guerra, 2004]. However, the sequences used here did not show any hybridization signals in the heterochromatic regions of $A$. aestiva. It is important to mention that $A$. aestiva exhibited heterochromatic blocks in its microchromosomes, a feature similar to what was described in some Cacatuidae like Cacatua roseicapilla and C. galerita, for example [Christidis et al., 1991].

Hybridization with telomeric sequences did not reveal ITSs in M. monachus. On the other hand, pair 2 and the $\mathrm{Z}$ chromosome of $A$. aestiva presented ITSs in the pericentromeric regions (Fig. 6A), which correspond to large heterochromatin blocks in both chromosomes, according to C-banding, probably because repetitive sequences of these regions are similar to telomeric ones, as proposed by Nanda et al. [2007], based on similar results observed in Nymphicus hollandicus.

\section{Evolution of Sex Chromosomes}

Another peculiarity of the M. monachus karyotype is that the $\mathrm{W}$ chromosome is similar in size and morphology to the Z. This is an uncommon feature in the Carinata group, with only a few exceptions like Neochmia phaeton (Passeriformes) [Christidis, 1986] and Nyctibius griseus (Caprimulgiformes) [Nieto et al., 2012]. Nonetheless, this scenario is common in ratite birds, in which both $\mathrm{Z}$ and $\mathrm{W}$ sex chromosomes are morphologically very similar in size [Ansari et al., 1988; Griffiths et al., 1998]. However, there is a notable difference between the W chromosome in M. monachus and in ratites by Zoo-FISH molecular data and C-banding.

Hybridization with the GGAZ probe shows little homology between $\mathrm{Z}$ and $\mathrm{W}$ chromosomes in M. monachus, since the corresponding probe did not produce any signal in the latter, being restricted to the $\mathrm{Z}$ chromosome. By contrast, in ratites, both $\mathrm{Z}$ and $\mathrm{W}$ were hybridized by GGAZ [Shetty et al., 1999], because both sex chromosomes are virtually homologous in ratites, representing a ZW sex system in an early stage of differentiation [Stiglec et al., 2007]. Additionally, in ratite birds (Rhea pennata and $R$. americana) constitutive heterochromatin in sex chromosomes is limited to the centromeric region and the short arms of the W chromosome [Pigozzi and Solari, 1997, 1999]. On the other hand, C-banding demonstrates that most of the W chromosome of M. monachus consists of constitutive heterochromatin, just like in the rest of Neognathae birds.

The use of microsatellite sequence probes provided evidence concerning the evolution of the sex chromosomes in Psittacidae. For instance, the sequence $(C G)_{n}$ was detected in the $\mathrm{Z}$ of both species, but in A. aestiva, it was present only in the telomeric regions, while in M. monachus, it was found interstitially in the long arms, confirming a possible amplification of this sequence. Moreover, M. monachus also presented blocks of the $(\mathrm{CAA})_{\mathrm{n}}$ and $(\mathrm{CAG})_{\mathrm{n}}$ microsatellite sequences on the $\mathrm{W}$ chromosome, different from the scenario observed in A. aestiva. In addition, the sequence $(C A G)_{n}$ was also widely distributed through the genome of $M$. monachus, generally corresponding to heterochromatin regions and microchromosomes (Fig. 4C, E). Hybridizations with GGAZ also revealed that a small region of one of the arms of the $\mathrm{Z}$ chromosome did not show a signal in both species. This could be due to the accumulation of repetitive sequences, even though no signal was detected with any of the microsatellite probes in this specific region.

So, taking into consideration the assumptions concerning the evolution of sex chromosomes, and consider- 
ing that Neognathae usually show a pattern in which the $\mathrm{W}$ chromosome is smaller than the $\mathrm{Z}$, we propose that the accumulation and amplification of repetitive sequences that occurred in the $\mathrm{W}$ chromosome of $M$. monachus led to the increase in its size. This corroborates the idea that, with lack of recombination, repetitive sequences are amplified in the nonrecombining region, resulting in the heterochromatinization of sex-specific chromosomes [Charlesworth et al., 1994; Schartl et al., 2016] (Fig. 7). Supporting this idea, recent studies of repetitive sequence mapping in Sauropsida (reptiles and birds) demonstrated that microsatellite amplification is associated with differentiation of sex chromosomes in different reptilian species [Matsubara et al., 2016].

This wide distribution of certain sequences can also be the result of transposable elements that have the capacity to copy and transpose themselves into nonhomologous regions in the genome [Kidwell, 2005; Bhargava and Fuentes, 2010]. These DNA segments play a crucial role in the genome evolution of several organisms [Biémont and Vieira, 2006], which could explain the wide distribution of the $(\mathrm{CAG})_{\mathrm{n}}$ sequence in M. monachus and the intense reorganization of its genome regarding other Psittacidae species. However, it is important to keep in mind the existence of other mechanisms that may act in the formation and expansion of microsatellite accumulation, like replication slippage and the insertion or deletion of one or more repeats [Levinson and Gutman, 1987; Ellegren, 2000].

In conclusion, the results obtained by mapping of microsatellite sequences, C-banding, and whole-chromo- some painting corroborate previous studies that show the importance of the accumulation/amplification of repetitive sequences in tandem in the morphological differentiation of sex chromosomes in birds, similar to what has been already well documented in other vertebrate groups. Further studies using next-generation sequencing data could bring a better elucidation about sex chromosome (ZW) evolution in birds.

\section{Acknowledgements}

The authors would like to thank IBAMA/SISBIO, Parque Zoobotânico Museu Paraense Emilio Goeldi (Belém, PA), and the group of UNIPAMPA for permission and collection of biological samples, CNPq (309699/2015-0) and Instituto Evandro Chagas (SVS/MS) for logistical and financial support. We would like to thank all colleagues of the Laboratory of Tissue Culture and Cytogenetics (SAMAM, Instituto Evandro chagas) for technical support.

\section{Statement of Ethics}

All experiments followed the standards approved by the Use of Animals for Research Ethics Committee (CEPAE-UFPA number 170-13).

\section{Disclosure Statement}

The authors declare no conflicts of interest.

\section{References}

Ansari H, Takagi N, Sasaki M: Morphological differentiation of sex chromosomes in three species of ratite birds. Cytogenet Cell Genet 47: 185-188 (1988).

Aquino R, Ferrari I: Chromosome study of Amazona amazonica and A. aestiva (Aves: Psittaciformes) determination of chromosome number and identification of sex chromosomes by C-banding methods. Genetica 81:1-3 (1990).

Bhargava A, Fuentes FF: Mutational dynamics of microsatellites. Mol Biotechnol 44:250-266 (2010).

Biémont C, Vieira C: Genetics: junk DNA as an evolutionary force. Nature 443:521-524 (2006).
Caparroz R, Duarte JMB: Chromosomal similarity between the scaly-headed parrot (Pionus maximiliani), the short-tailed parrot (Graydidascalus brachyurus) and the yellowfaced parrot (Salvatoria xanthops) (Psittaciformes: Aves): a cytotaxonomic analysis. Genet Mol Biol 27:522-528 (2004).

Charlesworth B, Sniegowski P, Stephan W: The evolutionary dynamics of repetitive DNA in eukaryotes. Nature 371:215-220 (1994).

Charlesworth D, Charlesworth B, Marais G: Steps in the evolution of heteromorphic sex chromosomes. Heredity 95:118-128 (2005).

Christidis L: Chromosomal evolution within the family Estrildidae (Aves) I: The Poephilae. Genetica 71:81-97 (1986).
Christidis L, Shaw DD, Schodde R: Chromosomal evolution in parrots, lorikeets and cockatoos (Aves: Psittaciformes). Hereditas 114: 47-56 (1991).

Cioffi MB, Bertollo LAC: Chromosomal distribution and evolution of repetitive DNAs in fish, in Garrido-Ramos MA (ed): Repetitive DNA. Genome Dyn 7:197-221 (2012).

Cioffi MB, Camacho JPM, Bertollo LAC: Repetitive DNAs and differentiation of sex chromosomes in neotropical fishes. Cytogenet Genome Res 132:188-194 (2011a).
Mapping of Repetitive DNAs in

M. monachus and A. aestiva
Cytogenet Genome Res 2017;151:151-160 DOI: $10.1159 / 000464458$ 
Cioffi MB, Kejnovsky E, Bertollo LAC: The chromosomal distribution of microsatellite repeats in the genome of the wolf fish Hoplias malabaricus, focusing on the sex chromosomes. Cytogenet Genome Res 132:289-296 (2011b).

de Lucca EJ, de Marcod A: Chromosomal polymorphism in Forpus xanthopterygius (Psittaciformes: Aves). Caryologia 36:355-361 (1983).

de Lucca EJ, Shirley LR, Lanier C: Karyotype studies in twenty-two species of parrots (Psittaciformes: Aves). Genet Mol Biol 14:73-98 (1991).

de Oliveira EHC, Habermann F, Lacerda O, Sbalqueiro IJ, Wienberg J, Müller S: Chromosome reshuffling in birds of prey: the karyotypes of the world's largest eagle (Harpy eagle, Harpia harpyja) compared to that of the chicken (Gallus gallus). Chromosoma 114: 338-343 (2005).

Duarte JMB, Caparroz R: Cytotaxonomic analysis of Brazilian species of the genus Amazona (Psittacidae, Aves) and confirmation of the genus Salvatoria (Ribeiro, 1920). Braz J Genet 18:623-628 (1995).

Duarte JMB, Giannoni ML: Karyotype of the little blue macaw Cyanopsitta spixii (Psittaciformes, Aves). Braz J Genet 13:137-140 (1990).

Ellegren H: Microsatellite mutations in the germline: implications for evolutionary inference. Trends Genet 16:551-558 (2000).

Ezaz T, Deakin JE: Repetitive sequence and sex chromosome evolution in vertebrates. Adv Evol Biol 2014:104683 (2014).

Ezaz T, Sarre S, O’Meally D, Graves JAM, Georges A: Sex chromosome evolution in lizards: independent origins and rapid transitions. Cytogenet Genome Res 127:249-260 (2009).

Ezaz T, Azad B, O’Meally D, Young MJ, Matsubara K, et al: Sequence and gene content of a large fragment of a lizard sex chromosome and evaluation of candidate sex differentiating gene R-spondin 1. BMC Genomics 14:899 (2013).

Francisco MR, Galetti PM Jr: Cytotaxonomic considerations on Neotropical Psittacidae birds and description of three new karyotypes. Hereditas 134:225-228 (2001).

Galetti PM Jr, Lima NRW, Venere PC: A monophyletic ZW sex chromosome system in Leporinus (Anostomidae, Characiformes). Cytologia 60:375-382 (1995).

Goldschmidt B, Nogueira DM, Monsores DW, Souza LM: Chromosome study in two Aratinga species (A. guarouba and A. acuticauda) (Psittaciformes). Braz J Genet 20:659-662 (1997).

Griffin DK, Haberman F, Masabanda J, O’Brien $\mathrm{P}$, Bagga M, et al: Micro- and macrochromosome paints generated by flow cytometry and microdissection: tools for mapping the chicken genome. Cytogenet Cell Genet 87:278-281 (1999).
Griffiths R, Double MC, Orr K, Dawson RJG: A DNA test to sex most birds. Mol Ecol 7:10711075 (1998).

Guerra M: Fish: Conceitos e aplicações na citogenética, pp 36-37 (Sociedade Brasileira de Genética, Ribeirão Preto 2004).

Hobza R, Lengerova M, Svoboda J, Kubekova H, Kejnovsky E, Vyskot B: An accumulation of tandem DNA repeats on the Y chromosome in Silene latifolia during early stages of sex chromosome evolution. Chromosoma 115: 376-382 (2006).

Hughes AL: Adaptive Evolution of Genes and Genomes (Oxford University Press, Oxford 1999).

Hughes AL, Hughes MK: Small genomes for better flyers. Nature 377:391 (1995).

Ijdo JW, Wells RA, Baldini A, Reeders ST: Improved telomere detection using a telomere repeat probe (TTAGGG)n generated by PCR. Nucleic Acids Res 19:4780 (1991).

Kejnovsky E, Hobza R, Cermak T, Kubat Z, Vyskot B: The role of repetitive DNA in structure and evolution of sex chromosomes in plants. Heredity 102:533-541 (2009).

Kidwell MG: Transposable elements, in Gregory TR (ed). The Evolution of the Genome, pp. 165-213 (Elsevier Academic Press, San Diego 2005)

Kraemer C, Schmidt ER: The sex determining region of Chironomus thummi is associated with highly repetitive DNA and transposable elements. Chromosoma 102:553-562 (1993).

Kubat Z, Hobza R, Vyskot B, Kejnovsky E: Microsatellite accumulation on the Y chromosome of Silene latifolia. Genome 51:350-356 (2008).

Laurance WF, Lovejoy TE, Vasconcelos HL, Bruna EM, Dirham RK, et al: Ecosystem decay of Amazonian forest fragments: a 22 -year investigation. Conserv Biol 16:605-618 (2002).

Levinson G, Gutman GA: Slipped-strand mispairing: a major mechanism for DNA sequence evolution. Mol Biol Evol 4:203-221 (1987).

Lunardi VO, Francisco MR, Rocha GT, Goldschmidt B, Galetti PM Jr: Karyotype description of two Neotropical Psittacidae species: the endangered hyacinth macaw, Anodorhynchus hyacinthinus, and the hawk-headed parrot, Deroptyus accipitrinus (Psittaciformes: Aves), and its significance for conservation plans. Genet Mol Biol 26:283-287 (2003).

Marchal JA, Acosta MJ, Bullejos M, Díaz R, Sánchez A: A repeat DNA sequence from the $Y$ chromosome in species of the genus Microtus. Chromosome Res 12:757-765 (2004).

Matsubara K, O’Meally D, Azad B, Georges A, Sarre SD, et al: Amplification of microsatellite repeat motifs is associated with the evolutionary differentiation and heterochromatinization of sex chromosomes in Sauropsida. Chromosoma 125:111-123 (2016).

Nanda I, Karl E, Griffin DK, Schartl M, Schmid M: Chromosome repatterning in three representative parrots (Psittaciformes) inferred from comparative chromosome painting. Cytogenet Genome Res 117:43-53 (2007).
Nieto LM, Kretschmer R, Ledesma MA, Garnero ADV, Gunski RJ: Karyotype morphology suggests that the Nyctibius griseus (Gmelin, 1789) carries an ancestral ZW-chromosome pair to the order Caprimulgiformes (Aves). Comp Cytogenet 6:379-387 (2012).

Pigozzi MI, Solari AJ: Extreme axial equalization and wide distribution of recombination nodules in the primitive ZW pair of Rhea americana (Aves, Ratitae). Chromosome Res 5: 421-428 (1997).

Pigozzi MI, Solari AJ: The ZW pairs of two paleognath birds from two orders show transitional stages of sex chromosome differentiation. Chromosome Res 7:541-551 (1999).

Presti FT, Wasko AP: A review of microsatellite markers and their application on genetic diversity studies in parrots. Open J Genet 4:6977 (2014).

Santos LP, Gunski RJ: Revisão de dados citogenéticos sobre a avifauna brasileira. Revista Brasileira de Ornitologia 14:35-45 (2006).

Sasaki M, Ikeuchi T, Maino S: A feather pulp culture for avian chromosomes with notes on the chromosomes of the peafowl and the ostrich. Experientia 24:1292-1293 (1968).

Schartl M, Schmid M, Nanda I: Dynamics of vertebrate sex chromosome evolution: from equal size to giants and dwarfs. Chromosoma 125:553-571 (2016).

Seabright M: A rapid banding technique for human chromosomes. Lancet 2:971-972 (1971).

Shetty S, Griffin DK, Graves JAM: Comparative painting reveals strong chromosome homology over 80 million years of bird evolution. Chromosome Res 7:289-295 (1999).

Stiglec R, Ezaz T, Graves JAM: A new look at the evolution of avian sex chromosomes. Cytogenet Genome Res 117:103-109 (2007).

Sumner AT: A simple technique for demonstrating centromeric heterochromatin. Exp Cell Res 75:304-306 (1972).

Van Dongen MWM, Boer LEM: Chromosome studies of 8 species of the families Cacatuidae and Psittacidae (Aves: Psittaciformes). Genetica 65:109-117 (1984).

Venton D: Highlight - small genomes for the swift: of flight and intron size. Genome Biol Evol 4:1044-1045 (2012).

Wachtel SS, Tiersch TR: Variations in genome mass. Comp Biochem Physiol B 104:207-213 (1993).

Wright TF, Toft CA, Enkerlin-Hoeflich E, Gonzalez-Elizondo J, Albornoz M, et al: Nest poaching in Neotropical parrots. Conserv Biol 15:710-720 (2001).

Zhang G, Li C, Li Q, Li B, Larkin DM, et al: Comparative genomics reveals insights into avian genome evolution and adaptation. Science 346:1311-1320 (2014).

Zhou Q, Zhang J, Bachtrog D, An N, Huang Q, et al: Complex evolutionary trajectories of sex chromosomes across bird taxa. Science 346:1332-1340 (2014).

de Oliveira Furo et al. 